\title{
No association of breast cancer risk with integrin betas (ITGB3) Leu33Pro genotype
}

\author{
SE Bojesen', A Tybjærg-Hansen ${ }^{2,3}$, CK Axelsson ${ }^{4}$ and BG Nordestgaard ${ }^{* 1,2}$ \\ 'Department of Clinical Biochemistry, Herlev University Hospital, Herlev Ringvej 75, Herlev DK-2730, Denmark; ${ }^{2}$ The Copenhagen City Heart Study, \\ Bispebjerg University Hospital, Bispebjerg Bakke 23, Copenhagen NV DK-2400, Denmark; ${ }^{3}$ Department of Clinical Biochemistry, Rigshospitalet, \\ Copenhagen University Hospital, Blegdamsvej 9, Copenhagen Ø DK-2 100, Denmark; ${ }^{4}$ Department of Breast Surgery, Herlev University Hospital, Herlev \\ Ringvej 75, Herlev DK-2730, Denmark
}

To pursue a borderline increased risk of breast cancer for carriers of two integrin beta3 (ITGB3) 33Pro alleles found in a recent prospective study, we conducted a case-control study of 1088 women with breast cancer and 4815 female controls. Leu33Pro heterozygotes, homozygotes and heterozygotes + homozygotes vs noncarriers had odds ratios for breast cancer of 1.0 ( $95 \%$ confidence interval: $0.8-1.1), 0.8(0.5-1.2)$ and I.0 (0.8- I.I), respectively. After stratification for conventional risk factors, odds ratio for breast cancer in heterozygotes, homozygotes and heterozygotes + homozygotes vs noncarriers were not increased above 1.0 in any of the 14 strata examined. This was also true after stratification for tumour histological subtype and cancer stage at the time of diagnosis.

British Journal of Cancer (2005) 93, 167-171. doi: I0.1038/sj.bjc.6602674 www.bjcancer.com

Published online 2 I June 2005

(C) 2005 Cancer Research UK

Keywords: integrins; breast cancer; genetic polymorphism; genetic epidemiology; case-control

In a recent large prospective study of the general population, we demonstrated an increased risk of all cancer among integrin beta $_{3}$ (ITGB3) Leu33Pro homozygotes (Bojesen et al, 2003). It is unclear as to which cancer subtype(s) account for this; however, subanalyses suggested that this risk increase partly could be caused by an increased risk of invasive breast cancer, ovarian cancer and/or melanoma. Although two later case-control studies did not confirm an increased risk of invasive breast cancer among Leu33Pro homozygotes (Ayala et al, 2003; Jin et al, 2004), certain considerations nevertheless favour the possibility that the ITGB3 Leu33Pro polymorphism may influence risk of invasive breast

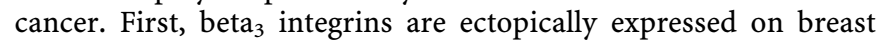
carcinoma cells where they enhance invasive and metastasiogenic properties of these cells (Murthy et al, 1996; Wewer et al, 1997; Chen et al, 2001, 2004; Rolli et al, 2003). Second, the Pro33 vs Leu33 allele enhances integrin-mediated activation of MAPK pathways (Vijayan et al, 2003), crucial for the malignant potential of cancer cells (Johnson and Lapadat, 2002). Third, in our previous prospective study there appeared to be a gene-dosage relationship as Leu33Pro heterozygotes and homozygotes vs noncarriers had hazard ratios for invasive breast cancer of 1.2 (95\% confidence interval: $0.9-1.7)$ and $1.9(1.0-3.7)$.

For this reason, we tested the hypothesis that ITGB3 Leu33Pro heterozygosity and homozygosity have an increased risk of invasive breast cancer. Given the prospective data, the assumption

\footnotetext{
* Correspondence: Professor BG Nordestgaard;

E-mail: brno@herlevhosp.kbhamt.dk

Received 4 March 2005; revised 4 May 2005; accepted 23 May 2005; published online 21 June 2005
}

of a dominant genetic effect seems reasonable, so we also combined the heterozygous and the homozygous participants in one group $v s$ noncarrier participants. We recruited 1088 consecutive female patients with invasive breast cancer and compared these with 4815 women from the general population without breast cancer. This large case-control study also allowed us to search for genotype-associated increased risk of breast cancer in women stratified for conventional risk factors, and to determine whether genotype-associated breast cancer risk is restricted to certain histological subtypes or cancer stages.

\section{MATERIALS AND METHODS}

\section{Study populations}

Cases of invasive breast cancer in women $(n=1088)$ were consecutively collected from the Department of Breast Surgery, Herlev University Hospital from 2001 to 2004. Owing to centralised breast cancer care, this department covers all cases of invasive breast cancer in roughly half of the Greater Copenhagen area, a population amounting to 320000 women the 1st of January 2004 . All patients filled in a questionnaire on lifestyle and health, including reproductive, menstrual and hormonal history, alcohol consumption, height and weight. Premenopausal women were defined as women who had a menstrual period within the previous 2 months. Postmenopausal women had a period more than 12 months ago. If the menstrual period was 2-12 months, the level of follicular-stimulating hormone decided whether the patient was pre- or postmenopausal. Furthermore, women $>55$ years of age after hysterectomy or with menstruation on hormonal substitution 
were considered postmenopausal. One or more questionnaire informations were missing for 546 women. However, for test of the main hypothesis (genotype association with risk of breast cancer adjusted for age), full information was available on all 1088 breast cancer cases.

Histological classification of tumours according to the WHO classification (Scarff and Torloni, 1968) and histological grading according to Bloom and Richardson (1957) was performed by pathologists blinded to patient genotype. Grade of malignancy of tumours with ductal histology was scored according to Scarff, Bloom and Richardson (Bloom and Richardson, 1957; Scarff and Torloni, 1968). Oestrogen and progesterone receptor status were determined with immunohistochemistry. An examination was positive if at least $10 \%$ of the nuclei in malignant tissue presented with typical staining for oestrogen and/or progesterone receptors. Owing to delay in registration, we did not have information on all these characteristics for 278 women.

The controls were women recruited from the other half of the Greater Copenhagen area from the 3rd examination (1991-94) of the Copenhagen City Heart Study (Appleyard et al, 1989; Schnohr et al, 2001). Age and other covariates used in the statistical models were recorded at this examination. Cases of breast cancer registered until the end of 2002 in the Danish Cancer Registry were excluded from the controls. In both cases and controls, $>97 \%$ were whites of Danish descent.

\section{Ethical approvals}

All participants gave written informed consent. The scientific ethical committee of Copenhagen and Frederiksberg approved the collection of controls (No. 100.2039/91), and the scientific ethical committee of the County of Copenhagen approved the collection of cases (KA 02152). The studies were also approved by Herlev University Hospital.

\section{Genotyping}

Participants were genotyped as described earlier (Zimrin et al, 1990). In short, the Leu33Pro polymorphism is a $\mathrm{T} \rightarrow \mathrm{C}$ substitution in exon 3 at position 176 in the beta ${ }_{3}$ integrin gene (GenBank Accession no. NM000212.1), which introduces an MspI recognition site. The assay also included a second MspI recognition site always cleaved, which served as a control site for the digestion reaction. A $268 \mathrm{bp}$ fragment of exon 3 was amplified from genomic DNA with flanking intronic primers, cleaved with $\mathrm{Msp}$ I, run on a $3 \%$ agarose gel and visualised by staining with ethidium bromide. Genotypes were determined independently by an experienced lab technician and an author (SEB).

\section{Statistical analysis}

The statistical software package STATA (2004) was used. All statistical tests were two-sided. $P<0.05$ was considered statistically significant. We used Mann - Whitney $U$-test and Pearson's $\chi^{2}$ test. Correction for multiple comparisons was by the Bonferoni method. We used NCSS 2001 and PASS 2000 power calculation software (Hintze, 2001) and a logistic regression power analysis to calculate the statistical power of this study.

We performed a matched case-control study with strata of 1,3 and 5 years of age. This resulted in 63 one-year strata with a mean of 4.5 controls per case, 22 three-year strata and 4.5 controls per case and 14 five-year strata and 4.6 controls per case, respectively. Using 1-year strata, two cases both aged 93 were unmatched with controls and 168 controls aged less than 28 were unmatched with cases. Conditional logistic regression was used to calculate odds ratio for invasive breast cancer according to genotype. Two models were applied: an age-matched model and an age-matched model also adjusted multifactorially for body mass index (continuous), alcohol consumption (continuous), parity (0vs more childbirths), use of oral contraceptive pill (yes/no), menopausal status (pre- $v s$ postmenopausal) and use of hormonal replacement therapy (yes/ no). In the analysis of histological subtype and invasive breast cancer stage, each subtype/stage was compared to the controls with the age-matched model of 1-year age strata while excluding cases without the analysed subtype/stage.

\section{RESULTS}

Characteristics of participants are shown in Table 1 . In cases and controls, $768(71 \%)$ and $3385(70 \%)$ were noncarriers, $296(27 \%)$ and $1300(27 \%)$ were heterozygotes and $24(2 \%)$ and $130(3 \%)$ were homozygotes, respectively. These genotype distributions were both in Hardy-Weinberg equilibrium $(P=0.67$ and 0.70$)$.

Age-matched odds ratios for breast cancer in heterozygotes, homozygotes and heterozygotes + homozygotes $v s$ noncarriers were 1.0 (95\% confident interval: $0.8-1.1), 0.8(0.5-1.2)$ and 1.0 $(0.8-1.1)$, respectively (Table 2$)$. Identical analyses with age strata of 3 and 5 years for matching of cases and controls gave similar results. Multifactorial adjustment did not substantially change these odds ratios. We had $90 \%$ statistical power to detect odds ratios equal to or larger than $1.3,1.8$ and 1.3 in heterozygous, homozygous and heterozygous + homozygous vs noncarriers, respectively.

To explore whether genotype interacts with conventional risk factors for breast cancer, we stratified the age-matched and multifactorially adjusted analyses by age, body mass index, alcohol consumption, parity, use of oral contraceptive pills, menopausal status and use of hormonal replacement therapy. Risk of breast cancer in homozygotes, heterozygotes and heterozygous + homozygous $v s$ noncarriers was not increased above 1.0 in any of the 14 subgroups of women examined (Figure 1). Although the adjusted odds ratio in homozygotes $v s$ noncarriers among women with alcohol consumption $>48$ g week $^{-1}$ was 0.3 $(0.1-0.9)$, this association became nonsignificant after correcting for multiple comparisons. Furthermore, this association was

Table I Characteristics of participants

\begin{tabular}{|c|c|c|c|}
\hline Number & 1088 & 4815 & - \\
\hline Age (years) & $62(29-93)$ & $61(22-90)$ & $<0.0001$ \\
\hline Alcohol consumption $\left(\right.$ g week $^{-1}$ ) & $48(0-720)$ & $36(0-960)$ & 0.0002 \\
\hline Nulliparous (\%) & $13(11-16)$ & $25(24-26)$ & $<0.0001$ \\
\hline Use of oral contraceptive pill (\%) & $3(1-4)$ & $4(4-5)$ & 0.02 \\
\hline Postmenopausal (\%) & $76(73-79)$ & $71(69-72)$ & 0.0009 \\
\hline
\end{tabular}

Values represent median (range) or frequencies (95\% confidence interval). P-values are for invasive breast cancer vs controls on Mann-Whitney U-test or Pearson's $\chi^{2}$ test. 
Table 2 Risk of invasive breast cancer by ITGB3 Leu33Pro genotype

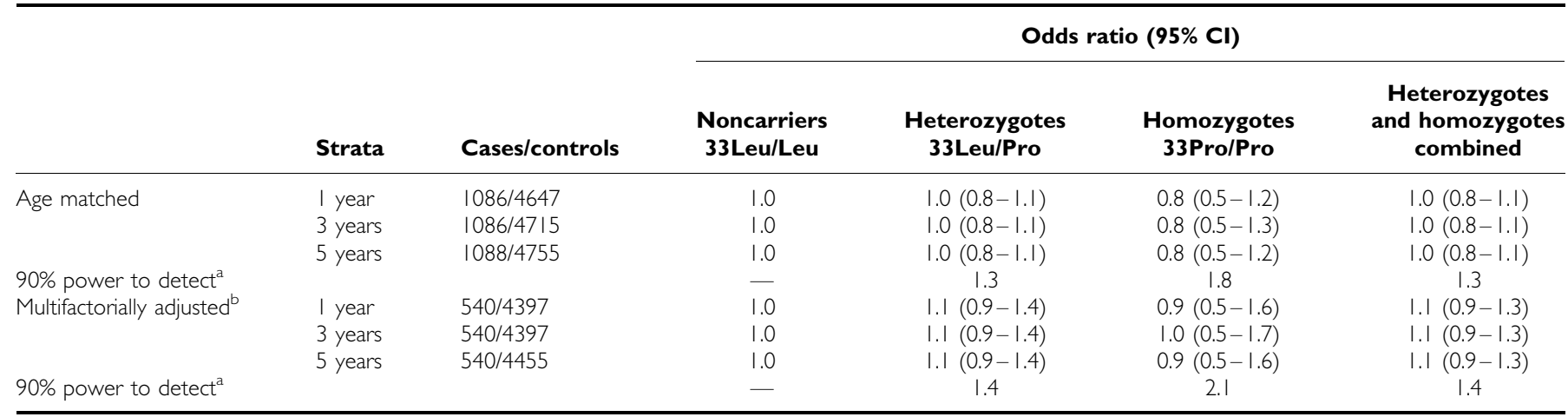

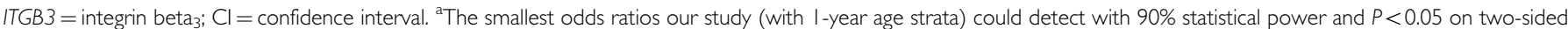
tests. 'Including body mass index (continuous), alcohol consumption (continuous), parity ( 0 vs more childbirths), use of oral contraceptive pill (yes/no), menopausal status (pre- vs postmenopausal), use of hormone replacement therapy (yes/no).

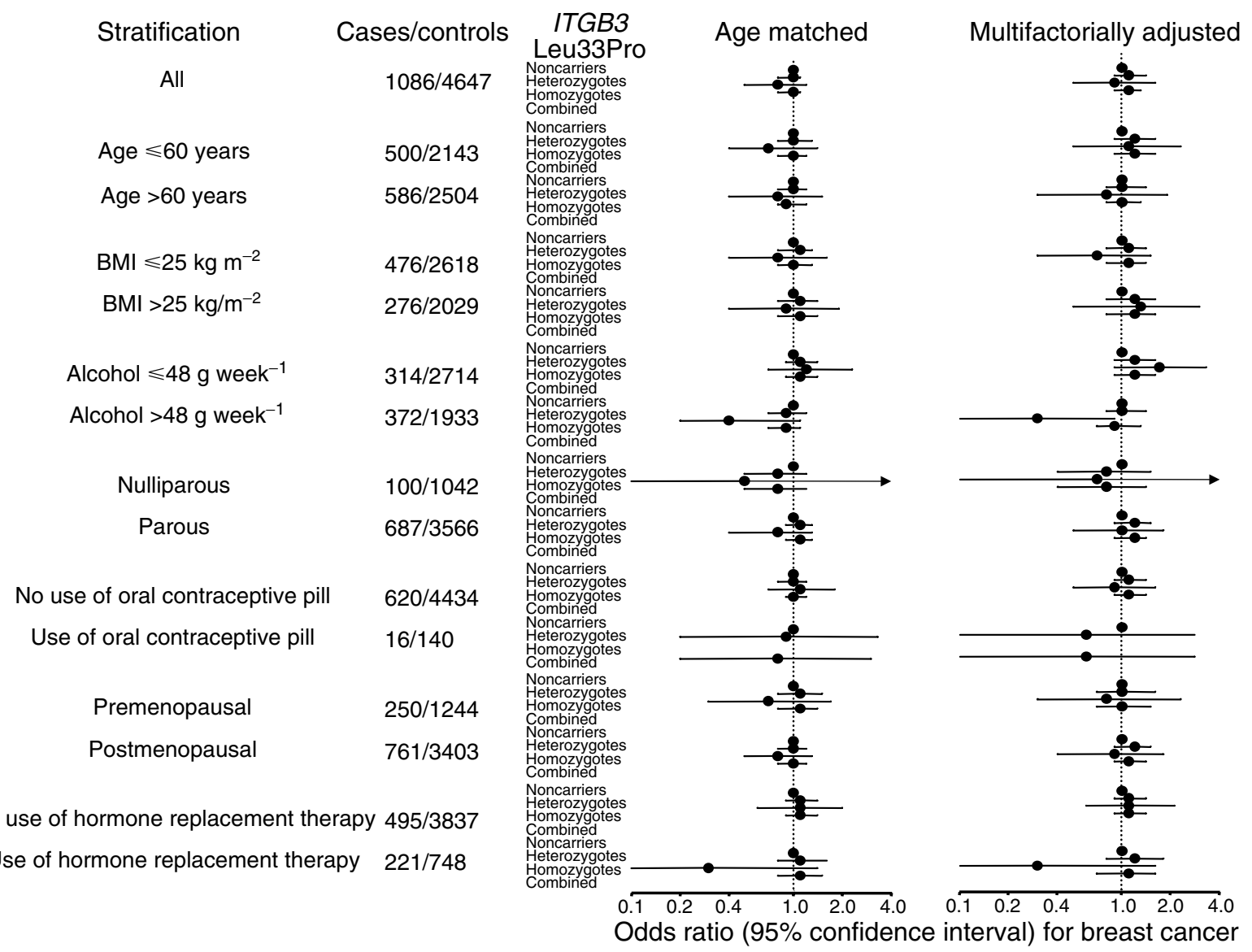

Figure I Risk of invasive breast cancer by ITGB3 Leu33Pro genotype. Number of cases/controls refer to the I-year age strata calculations and vary through stratifications due to incomplete information on the stratification in question. Multifactorial adjustment was for body mass index (continuous), alcohol consumption (continuous), parity ( 0 vs more childbirths), use of oral contraceptive pill (yes/no), menopausal status (pre- vs postmenopausal) and use of hormone replacement therapy (yes/no).

statistically nonsignificant in the age-matched study with more statistical power.

Risk of breast cancer in homozygotes, heterozygotes and heterozygous + homozygous vs noncarriers did not differ from 1.0 in any histological subgroup of invasive breast cancer Table 3.

Risk of invasive breast cancer in homozygotes, heterozygotes and heterozygous + homozygous vs non-carriers did not differ from 1.0 in any cancer stage subgroup (Table 4).

\section{DISCUSSION}

So far, four papers have been published on this polymorphism and breast cancer risk (Ayala et al, 2003; Bojesen et al, 2003; Jin et al, 2004; Wang-Gohrke and Chang-Claude, 2004). Our own first study was explorative in nature and found a hazard ratio in heterozygotes and homozygotes $v s$ noncarriers of $1.2(0.9-1.6)$ and 1.9 (1.0-3.7), $P$-value 0.06 , so this could represent a chance finding. In 
Table 3 Risk of invasive breast cancer by ITGB3 Leu33Pro genotype stratified for histological subtype

\begin{tabular}{|c|c|c|c|c|c|c|c|c|}
\hline $\begin{array}{l}\text { Histological } \\
\text { subtype }\end{array}$ & \multicolumn{2}{|c|}{ Noncarriers 33Leu/Leu } & \multicolumn{2}{|c|}{ Heterozygotes 33Leu/Pro } & \multicolumn{2}{|c|}{ Homozygotes 33Pro/Pro } & \multicolumn{2}{|c|}{ Heterozygotes and homozygotes combined } \\
\hline Lobular & 72 (68) & 1.0 & $32(30)$ & $1.2(0.8-1.8)$ & $2(2)$ & $0.7(0.2-2.9)$ & $34(32)$ & $1.1(0.7-1.7)$ \\
\hline Others & $37(76)$ & 1.0 & $12(24)$ & $0.8(0.4-1.6)$ & $0(0)$ & - & $12(24)$ & $0.8(0.4-1.5)$ \\
\hline Unknown & $202(72)$ & 1.0 & $70(25)$ & $0.9(0.7-1.2)$ & $8(3)$ & $0.9(0.4-2.0)$ & $78(28)$ & $0.9(0.7-1.2)$ \\
\hline
\end{tabular}

ITGB3 = integrin beta $; \mathrm{Cl}=$ confidence interval. Odds ratios were vs 4647 controls and matched for age in one-year strata.

Table 4 Risk of invasive breast cancer by ITGB3 Leu33Pro genotype stratified for characteristics of tumour or dissemination at diagnosis

\begin{tabular}{|c|c|c|c|c|c|c|c|c|}
\hline & \multicolumn{2}{|c|}{ Noncarriers 33Leu/Leu } & \multicolumn{2}{|c|}{$\begin{array}{l}\text { Heterozygotes 33Leu/ } \\
\text { Pro }\end{array}$} & \multicolumn{2}{|c|}{ Homozygotes 33Pro/Pro } & \multicolumn{2}{|c|}{$\begin{array}{c}\text { Heterozygotes and } \\
\text { homozygotes combined }\end{array}$} \\
\hline & $n(\%)$ & Odds ratio & $n(\%)$ & $\begin{array}{l}\text { Odds ratio } \\
(95 \% \mathrm{Cl})\end{array}$ & $n(\%)$ & $\begin{array}{l}\text { Odds ratio } \\
(95 \% \mathrm{Cl})\end{array}$ & $n(\%)$ & $\begin{array}{c}\text { Odds ratio } \\
\text { (95\%Cl) }\end{array}$ \\
\hline \multicolumn{9}{|l|}{ Tumour size (mm) } \\
\hline$\leqslant 10$ & $99(70)$ & 1.0 & $43(29)$ & I.I (0.8-1.6) & I (I) & $0.2(0.0-1.7)$ & $44(31)$ & $1.0(0.7-1.4)$ \\
\hline $\mid 1-20$ & $217(68)$ & 1.0 & $95(30)$ & $1.1(0.9-1.4)$ & $5(2)$ & $0.6(0.2-1.4)$ & $100(32)$ & $1.1(0.8-1.4)$ \\
\hline $21-30$ & $163(73)$ & 1.0 & $53(24)$ & $0.8(0.6-1.2)$ & $8(4)$ & $1.2(0.6-2.5)$ & $61(27)$ & $0.9(0.6-1.2)$ \\
\hline $31-50$ & $62(68)$ & 1.0 & $27(30)$ & $1.2(0.7-1.9)$ & $2(2)$ & $0.9(0.2-3.6)$ & $29(32)$ & $1.2(0.7-1.8)$ \\
\hline$>50$ & $26(76)$ & 1.0 & $8(24)$ & $0.8(0.4-1.9)$ & $0(0)$ & - & $8(24)$ & $0.8(0.3-1.7)$ \\
\hline Unknown & $201(73)$ & 1.0 & $68(25)$ & $0.9(0.7-1.2)$ & $8(3)$ & $0.9(0.4-2.0)$ & $76(27)$ & $0.9(0.7-1.2)$ \\
\hline \multicolumn{9}{|c|}{ Grade of malignancy (only ductal histology) } \\
\hline Grade I & $109(66)$ & 1.0 & $52(31)$ & $1.2(0.8-1.7)$ & $5(3)$ & I.I (0.4-2.7) & $57(34)$ & $1.2(0.8-1.6)$ \\
\hline Grade II & $226(72)$ & 1.0 & $83(26)$ & $1.0(0.7-1.2)$ & $6(2)$ & $0.7(0.3-1.5)$ & $89(28)$ & $0.9(0.7-1.2)$ \\
\hline Grade III & $110(72)$ & 1.0 & $40(26)$ & $0.9(0.7-1.4)$ & $3(2)$ & $0.6(0.2-2.0)$ & $37(27)$ & $0.9(0.6-1.3)$ \\
\hline Unknown & $12(71)$ & 1.0 & $5(29)$ & I.I $(0.4-3.1)$ & $0(0)$ & - & $5(29)$ & $0.9(0.3-2.7)$ \\
\hline \multicolumn{9}{|l|}{ Hormone receptor status } \\
\hline Negative & $95(7 \mathrm{I})$ & 1.0 & $36(27)$ & $1.0(0.7-1.5)$ & $2(2)$ & $0.4(0.1-1.7)$ & $38(29)$ & $0.9(0.6-1.4)$ \\
\hline Positive & 449 (69) & 1.0 & $186(29)$ & $1.1(0.9-1.3)$ & $13(2)$ & $0.7(0.4-1.3)$ & $199(31)$ & $1.0(0.9-1.2)$ \\
\hline Unknown & $224(73)$ & 1.0 & $72(24)$ & $0.8(0.6-1.1)$ & $9(3)$ & $1.0(0.5-2.0)$ & $81(27)$ & $0.8(0.6-1.1)$ \\
\hline \multicolumn{9}{|l|}{ Lymph node involvement } \\
\hline No tumour positive nodes & $278(7 I)$ & 1.0 & $104(26)$ & $1.0(0.8-1.2)$ & I| (3) & $1.0(0.5-1.8)$ & II5 (29) & $1.0(0.8-1.2)$ \\
\hline $\begin{array}{l}\text { Tumour-positive nodes, without } \\
\text { breakthrough of capsule }\end{array}$ & $154(73)$ & 1.0 & $56(26)$ & $0.9(0.7-1.3)$ & $2(1)$ & $0.3(0.1-1.2)$ & $58(27)$ & $0.9(0.6-1.2)$ \\
\hline $\begin{array}{l}\text { Tumour-positive nodes, with } \\
\text { breakthrough of capsule }\end{array}$ & $128(67)$ & 1.0 & $61(32)$ & $1.2(0.9-1.7)$ & $3(2)$ & $0.6(0.2-1.9)$ & $64(33)$ & $1.2(0.9-1.6)$ \\
\hline Unknown & $208(72)$ & 1.0 & $73(25)$ & $0.9(0.7-1.2)$ & $8(3)$ & $0.9(0.4-1.9)$ & $81(28)$ & $0.9(0.7-1.2)$ \\
\hline \multicolumn{9}{|l|}{ Distant metastases } \\
\hline Absent & $535(70)$ & 1.0 & $212(28)$ & $1.0(0.9-1.2)$ & $16(2)$ & $0.7(0.4-1.2)$ & $228(30)$ & $1.0(0.8-1.2)$ \\
\hline Present & $4(67)$ & 1.0 & $2(33)$ & $1.3(0.2-7.3)$ & $0(0)$ & - & $2(33)$ & $1.2(0.2-6.7)$ \\
\hline Unknown & $229(72)$ & 1.0 & $80(25)$ & $0.9(0.7-1.2)$ & $8(3)$ & $0.8(0.4-1.7)$ & $88(28)$ & $0.9(0.7-1.1)$ \\
\hline
\end{tabular}

ITGB3 = integrin beta $; \mathrm{Cl}=$ confidence interval. Odds ratios were vs 4647 controls and matched for age in one-year strata.

contrast to another study, Ayala et al (2003) reduced risk of breast cancer in heterozygotes $v s$ noncarriers in a case-control study of 100 cases and 100 controls. However, genotype distribution among controls was not in Hardy-Weinberg equilibrium $(P=0.048)$ and the finding was only significant at $P=0.04$, so this also could represent a chance finding. In a third study (Jin et al, 2004), no increased (or decreased) risk was found in heterozygotes or homozygotes. In the fourth study (Wang-Gohrke and ChangClaude, 2004) of 602 cases and 1054 controls, the odds ratios for heterozygotes, homozygotes and heterozygotes + homozygotes $v \mathrm{~s}$ noncarriers were $1.1(0.9-1.4), 1.3(0.7-2.4)$ and $1.1(0.9-1.4)$, respectively. A borderline $(P=0.055)$ interaction between age (age $\leqslant 45 v s>45$ years) and genotype (noncarriers $v s$ heterozygotes + homozygotes) with an odds ratio of heterozygotes + homozygotes $v s$ noncarriers of $1.3(1.0-1.8)$ in women aged over 44 years; in our study, no such interaction could be detected $(P=0.64)$. They also detected no association between tumour stage or grade and genotype (noncarrier $v s$ heterozygous + homozygous state) after correcting for multiple comparisons.

Like the previous studies mentioned, the present study has certain limitations. Covariates for controls were recorded at the 1991-1994 examination, while those for the cases were recorded at the time of diagnosis in 2001-2004, approximately 10 years apart. For conventional risk factors such as the use of hormonal replacement therapy, this could distort risk estimations, but will not invalidate analyses of genetic risk factors, as genotypes are permanent. Although cases and controls were recruited from slightly different geographical locations of the greater Copenhagen area, the background population is the same, more than $97 \%$ being white women of Danish descent in both cases and controls. 
Overall, the combined evidence from this and previous reports indicates that ITGB3 Leu33Pro heterozygosity and homozygosity do not increase the risk of breast cancer.

Future research should no longer focus on risk of breast cancer in ITGB3 Leu33Pro homozygotes, but should rather try to examine other cancer subgroups like ovarian cancer, melanoma or yet other cancer subgroups (Bojesen et al, 2003), as explanations for the increased overall cancer risk in Leu33Pro homozygotes.

\section{REFERENCES}

Appleyard M, Tybjærg-Hansen A, Jensen G, Schnohr P, Nyboe J (1989) The Copenhagen City Heart Study. Østerbroundersøgelsen. A book of tables with data from the first examination (1976 - 78) and a five year follow-up (1981-1983). Scand J Soc Med 170(Suppl 41): 1-160

Ayala F, Corral J, Gonzalez-Conejero R, Sanchez I, Moraleda JM, Vicente V (2003) Genetic polymorphisms of platelet adhesive molecules: association with breast cancer risk and clinical presentation. Breast Cancer Res Treat 80: 145 -154, Pubmed\# indexed for MEDLINE: PM:12908817

Bloom HJ, Richardson WW (1957) Histological grading and prognosis in breast cancer. Br J Cancer 11: 359-377

Bojesen SE, Tybjærg-Hansen A, Nordestgaard BG (2003) Integrin beta3 Leu33Pro homozygosity and risk of cancer. J Natl Cancer Inst 95: 11501157, Pubmed\# indexed for MEDLINE: PM:12902444

Chen J, Baskerville C, Han Q, Pan ZK, Huang S (2001) Alpha(v) integrin, p38 mitogen-activated protein kinase, and urokinase plasminogen activator are functionally linked in invasive breast cancer cells. $J$ Biol Chem 276: 47901 - 47905, Pubmed\# indexed for MEDLINE: PM:11606583

Chen P, Shen WZ, Karnik P (2004) Suppression of malignant growth of human breast cancer cells by ectopic expression of integrin-linked kinase. Int J Cancer 111: 881-891, Pubmed\# indexed for MEDLINE: PM: 15300800

Hintze J (2001) NCSS 2001 and PASS 2000. (Standard). USA: Number Cruncher Statistical Systems

Jin Q, Hemminki K, Grzybowska E, Klaes R, Soderberg M, Forsti A (2004) Re: Integrin beta3 Leu33Pro homozygosity and risk of cancer. J Natl Cancer Inst 96: 234-235, Pubmed\# indexed for MEDLINE: PM:14759991

Johnson GL, Lapadat R (2002) Mitogen-activated protein kinase pathways mediated by ERK, JNK, and p38 protein kinases. Science 298: 1911 -1912, Pubmed\# indexed for MEDLINE: PM:12471242

Murthy MS, Reid Jr SE, Yang XF, Scanlon EP (1996) The potential role of integrin receptor subunits in the formation of local recurrence and

\section{ACKNOWLEDGEMENTS}

We thank Hanne Damm and Mette Refstrup for excellent technical assistance and the breast cancer patients and the participants of the Copenhagen City Heart Study for their willingness to participate. This work was supported by the Danish Heart Foundation (ATH, BGN), Overlæge Johan Boserup og Lise Boserups Fond (BGN) and The Danish Medical Research Council (BGN).

distant metastasis by mouse breast cancer cells. J Surg Oncol 63: 77-86, Pubmed\# indexed for MEDLINE: PM:8888798

Rolli M, Fransvea E, Pilch J, Saven A, Felding-Habermann B (2003) Activated integrin alphavbeta3 cooperates with metalloproteinase MMP-9 in regulating migration of metastatic breast cancer cells. Proc Natl Acad Sci USA 100: 9482-9487, Pubmed\# indexed for MEDLINE: PM:12874388

Scarff RW, Torloni H (1968) Histological typing of breast tumors. In International Classification of Tumours, Sobin LH (ed) pp 13-20. Geneva: World Health Organization

Schnohr P, Jensen G, Lange P, Scharling H, Appleyard M (2001) The Copenhagen City Heart Study. Østerbroundersøgelsen, tables with data from the third examination 1991-1994. Eur Heart J 3: H1-H83

STATA SE (2004) STATA SE for Windows, rel. 8.2. (Standard). USA: StataCorp

Vijayan KV, Liu Y, Dong JF, Bray PF (2003) Enhanced activation of mitogen-activated protein kinase and myosin light chain kinase by the Pro33 polymorphism of integrin beta 3. J Biol Chem 278: 3860-3867, Pubmed\# indexed for MEDLINE: PM:12460991

Wang-Gohrke S, Chang-Claude J (2004) Integrin beta3 Leu33Pro polymorphism and breast cancer risk: a population-based case-control study in Germany. Breast Cancer Res Treat 88: 231-237, Pubmed\# indexed for MEDLINE: PM:15609125

Wewer UM, Shaw LM, Albrechtsen R, Mercurio AM (1997) The integrin alpha 6 beta 1 promotes the survival of metastatic human breast carcinoma cells in mice. Am J Pathol 151: $1191-1198$, Pubmed\# indexed for MEDLINE: PM:9358743

Zimrin AB, Gidwitz S, Lord S, Schwartz E, Bennett JS, White GC, Poncz M (1990) The genomic organization of platelet glycoprotein IIIa. J Biol Chem 265: 8590-8595, Pubmed\# indexed for MEDLINE: PM:2341395 